\title{
Survey on lower urinary tract symptoms and sleep disorders in patients treated at urology departments
}

This article was published in the following Dove Press journal:

Nature and Science of Sleep

25 January 2013

Number of times this article has been viewed

\author{
Nobutaka Shimizu' \\ Yasuharu Nagai' \\ Yutaka Yamamoto' \\ Takafumi Minami' \\ Taiji Hayashi' \\ Hidenori Tsuji' \\ Masahiro Nozawa' \\ Kazuhiro Yoshimura' \\ Tokumi Ishii' \\ Hirotsugu Uemura' \\ Takashi Oki² \\ Koichi Sugimoto ${ }^{2}$ \\ Kazuhiro Nose ${ }^{2}$ \\ Tsukasa Nishioka ${ }^{2}$ \\ 'Department of Urology, Kinki \\ University Faculty of Medicine, \\ Osaka, Japan; ${ }^{2}$ Department of Urology, \\ Sakai Hospital, Kinki University \\ Faculty of Medicine, Osaka, Japan
}

Correspondence: Nobutaka Shimizu Department of Urology, Kinki University Faculty of Medicine, 377-2 Ohno-Higashi, Osaka-Sayama, Osaka 589-85II, Japan

Tel +81723660221 ext 3524

Fax +81723656273

Email shimizun@med.kindai.ac.jp
Objectives: This study examined the association between sleep disorders and lower urinary tract symptoms in patients who had visited urology departments.

Methods: This was an independent cross-sectional, observational study. Outpatients who had visited the urology departments at the Kinki University School of Medicine or the Sakai Hospital, Kinki University School of Medicine, between August 2011 and January 2012 were assessed using the Athens Insomnia Scale and the International Prostate Symptom Score.

Results: In total, 1174 patients (mean age, $65.7 \pm 13.7$ years), with 895 men $(67.1 \pm 13.2$ years old) and 279 women (61.4 \pm 14.6 years old), were included in the study. Approximately half of these patients were suspected of having a sleep disorder. With regard to the International Prostate Symptom Score subscores, a significant increase in the risk for suspected sleep disorders was observed among patients with a post-micturition symptom (the feeling of incomplete emptying) subscore of $\geq 1$ (a 2.3-fold increase), a storage symptom (daytime frequency + urgency + nocturia) subscore of $\geq 5$ (a 2.7 -fold increase), a voiding symptom (intermittency + slow stream + hesitancy) subscore of $\geq 2$ (a 2.6-fold increase), and a nocturia subscore of $\geq 2$ (a 1.9-fold increase).

Conclusion: The results demonstrated that the risk factors for sleep disorders could also include voiding, post-micturition, and storage symptoms, in addition to nocturia.

Keywords: lower urinary tract symptoms, sleep disturbance, urological disease

\section{Introduction}

It has been indicated that in the present Japanese society, which is shifting to a "24-hour society" with a rapidly progressing aging population, one in five people complain of a sleep disorder. ${ }^{1}$ Sleep disorders interfere with people's social activities and quality of life (QOL); further, they act as risk factors for the development of lifestyle-related diseases such as hypertension, diabetes, and hyperlipidemia. ${ }^{2}$ In addition, sleep disorders have been closely associated with nocturia, ${ }^{3-9}$ where the percentage of individuals who void three or more times at night increases among those aged $\geq 60$ years; this is observed in approximately $30 \%$ of men and $20 \%$ of women who are aged $\geq 70$ years. $^{10}$ Moreover, some studies have reported that balance disorders (staggering) among individuals who rise to urinate at night could lead to increased mortality due to the associated risk of falling. ${ }^{11,12}$

Therefore, sleep disorders represent a major concern in the present 24-hour Japanese society that is witnessing an aging population. Consequently, it is likely that even nonspecialists in sleep disorders will be increasingly required to examine patients suffering from insomnia. In addition, given that lower urinary tract symptoms (LUTS) and sleep 
disorders deteriorate as people age, the authors reasoned that studying the association between LUTS and sleep disorders would provide important information in considering treatment options, thereby contributing to the improvement of patients' QOL. However, with the exception of nocturia, few reports have been published on the association between LUTS and sleep disorders among patients who receive outpatient urological treatment. Therefore, in this study, we have investigated the association between LUTS and sleep disorders, and in patients who visited urology departments for treatment. Further, this study aims to facilitate the early detection of insomnia.

\section{Methods}

This was an independent cross-sectional, observational study. A questionnaire was administered among 1174 outpatients who visited the Department of Urology, Kinki University School of Medicine, or the Department of Urology, Sakai Hospital, Kinki University School of Medicine between August 2011 and January 2012, and who were capable of understanding the interview sheets on their own. The 1174 subjects (mean age: $65.7 \pm 14.6$ years) consisted of 895 males $(67.1 \pm 13.2$ years $)$ and 279 females $(61.4 \pm 14.6$ years). A total of 746 patients were 65 years of age and over, and 428 were less than 65 years.

The questionnaire was approved by the Ethics Committee of Kinki University, and consent was obtained from patients before the onset of the study.

\section{Sleep disorders}

The questionnaire-based evaluation of sleep disorders considered whether or not the patient had used a sleep inducer, and also considered the patient's score on the Athens Insomnia Scale (AIS) (scores $<4$ indicated no suspicion of sleep disorders; scores $=4-5$ indicated some suspicion of sleep disorders; and scores $\geq 6$ indicated suspicion of sleep disorders), ${ }^{13,14}$ which was established by the World Health Organization Worldwide Project on Sleep and Health. The first screening was conducted on the AIS, and then the International Classification of Sleep Disorders, second edition ${ }^{15}$ was used to assess: (1) subjective insomnia; (2) the presence of insomnia in an environment suited for sleep; and (3) functional impairments during the daytime (eg, daytime sleepiness, as well as impaired attentive abilities and concentration).

\section{LUTS}

LUTS were evaluated using the International Prostate Symptom Score (IPSS).
The IPSS is an eight-question (seven questions on symptoms, and one question on QOL) written screening tool used to screen for, rapidly diagnose, track the symptoms of, and propose the management of benign prostatic hyperplasia (BPH). Presently, it is being used to evaluate LUTS associated with BPH as well as a variety of other diseases. The seven questions on symptoms are related to the urinary system and include feelings of incomplete emptying of the bladder, daytime frequency of urination, intermittency of urination, urgency to urinate, weak urinary stream, difficulty urinating, and nocturia. These questions referred to the period during the last month, and each question was assigned a score from 1 to 5 for a maximum of 35 points. The eighth question on the QOL was assigned a score from 1 to 6 .

\section{QOL}

QOL was evaluated according to the patients' current satisfaction level (0, very satisfied; 1 , satisfied; 2 , mostly satisfied; 3, uncertain; 4, somewhat dissatisfied; 5, dissatisfied; and 6 , very dissatisfied) in order to assess the association of sleep disorders and LUTS.

\section{Other urological diseases}

The definition of these diseases, including that for overactive bladder (OAB) symptoms, mainly remained the same prior to and during treatment, as well as during follow-up. OAB patients were defined as those with a pretreatment OAB symptom score of $\geq 2$ on the urgency subscale, and with an overall score of $\geq 3$. BPH patients were defined as those with an IPSS $\geq 8$, a prostate size $\geq 20 \mathrm{~g}$, and a maximum flow rate of $\leq 10 \mathrm{~mL} / \mathrm{second}$. Prostate cancer patients were defined as those with a histological diagnosis of prostate cancer using needle biopsy. Bladder cancer patients were defined as those who had a tumor confirmed prior to treatment using cystoscopy, and those who had been confirmed histologically during treatment and at follow-up.

Significant factors selected by univariate analysis were assessed by multivariate analysis using the multiple logistic regression model in order to analyze suspected sleep disorders as well as related factors. Suspected sleep disorders were characterized by the use of a sleep inducer, an AIS score of $\geq 6$, and AIS scores of 4-5. The Statistical Package for the Social Sciences version 20.0 (IBM Corporation, Armonk, NY, USA) was used for statistical analysis.

\section{Results}

Figure 1 illustrates the demographics of the 1174 subjects. The most common primary disease was prostatic hyperplasia, 


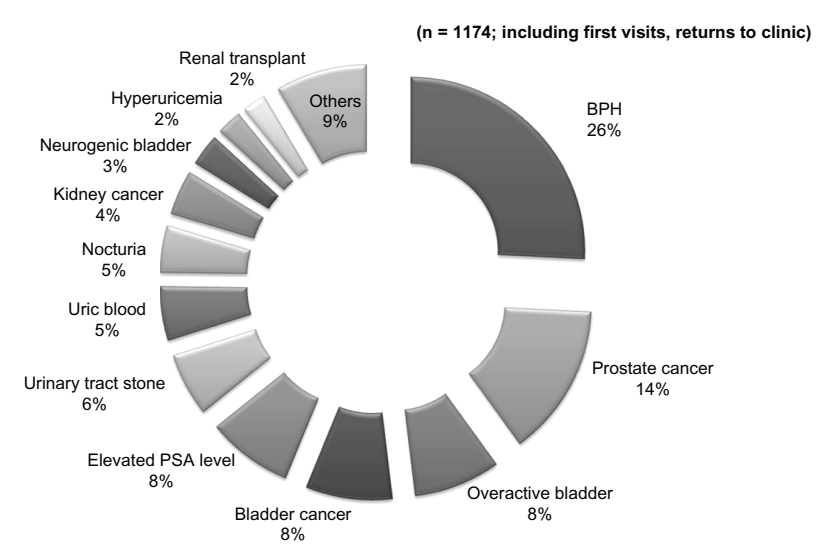

Figure I Diagnosis distribution.

Abbreviations: BPH, benign prostatic hyperplasia; PSA, prostate-specific antigen.

which was observed in 335 patients (25.8\%), followed by prostate cancer in 185 patients (14.2\%), OAB in 105 patients (8.1\%), bladder cancer in 105 patients (8.1\%), and elevated prostate-specific antigen levels in 104 patients $(8.0 \%)$.

\section{The AIS}

Overall, 197 subjects (16.8\%) were using sleep inducers; 217 subjects $(18.5 \%)$ scored $\geq 6$ on the AIS, $190(16.2 \%)$ scored 4-5, and $570(48.5 \%)$ scored $<4$ (Figure 2).

The suspected cases of sleep disorders are classified in Figure 3 into 10-year age groups. Patients on sleep inducers accounted for $7.1 \%$ of those aged $\leq 49$ years, and their percentage increased to $21.8 \%$ among patients aged $\geq 80$ years. If patients suspected of having a sleep disorder (ie, an AIS score of $4-5$ and $\geq 6$ ) were combined with those on sleep inducers, then they constituted more than $40 \%$ of patients across all age groups $(45.7 \%$ of patients aged $\leq 49$ years, and $49.6 \%$ of patients aged $\geq 80$ years).

\section{IPSS total score}

Based on the IPSS total scores, this risk significantly increased by 2.3 -fold among patients with moderate symptoms (scores of 8-19) and by 4.8 -fold among those with severe symptoms (scores of $\geq 20$ ), compared to patients with mild symptoms (scores of 0-7), as shown in Figure 4.

\section{IPSS subscores}

An increased risk of suspected sleep disorders was observed among patients with a post-micturition symptom (the feeling of incomplete emptying) subscore of $\geq 1$ (a 2.3-fold increase), a storage symptom (daytime frequency + urgency + nocturia) subscore of $\geq 5$ (a 2.7-fold increase), a voiding symptom (intermittency + slow stream + hesitancy) subscore of $\geq 2$ (a 2.6-fold increase), and a nocturia subscore of $\geq 2$ (a 1.9fold increase), as presented in Figure 5.

The odds ratio per unit subscore change was calculated at 1.3 for patients with post-micturition symptoms, 1.2 for those with storage symptoms, 1.1 for those with voiding symptoms, and 1.3 for those with nocturia symptoms (Figure 6). The Spearman's rank correlation coefficient suggested a positive correlation between nocturia and post-micturition, storage, and voiding symptom subscores $(P<0.001)$.

\section{Association between IPSS, QOL, and suspected sleep disorders}

The risk for suspected sleep disorders significantly increased by 3.3 -fold with a QOL score of $\geq 4$, and the

( $\mathrm{n}=1174$; including first visits, returns to clinic)

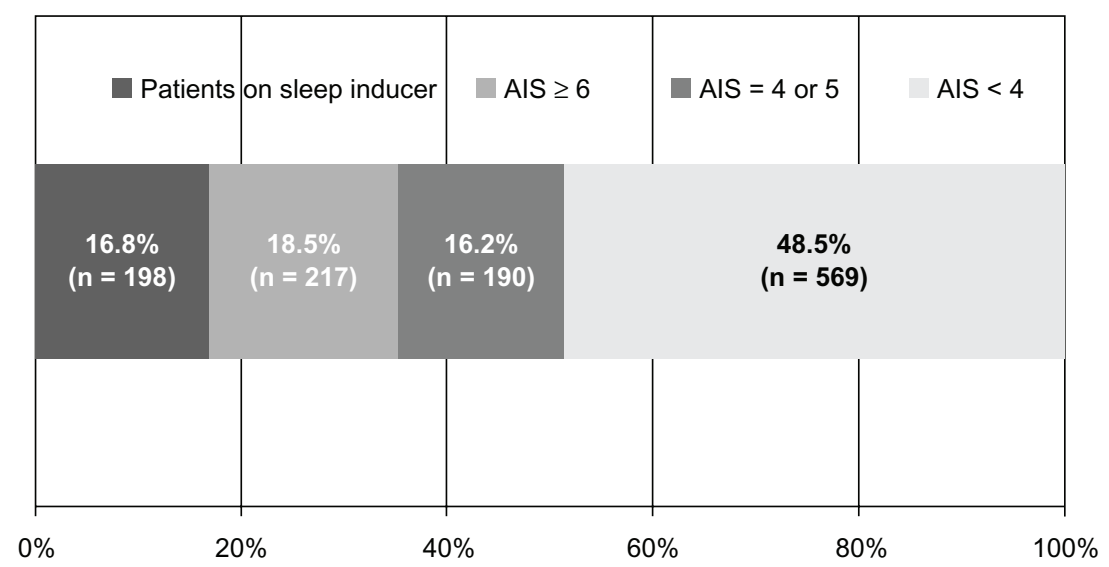

Figure 2 AIS.

Notes: *Classified according to the total AIS. AIS $<4$, no suspicion of sleep disorders; AIS $=4$ or 5 , some suspicion of sleep disorders; AIS $\geq 6$, suspicion of sleep disorders.

Abbreviations: AIS, Athens Insomnia Scale; n, number. 


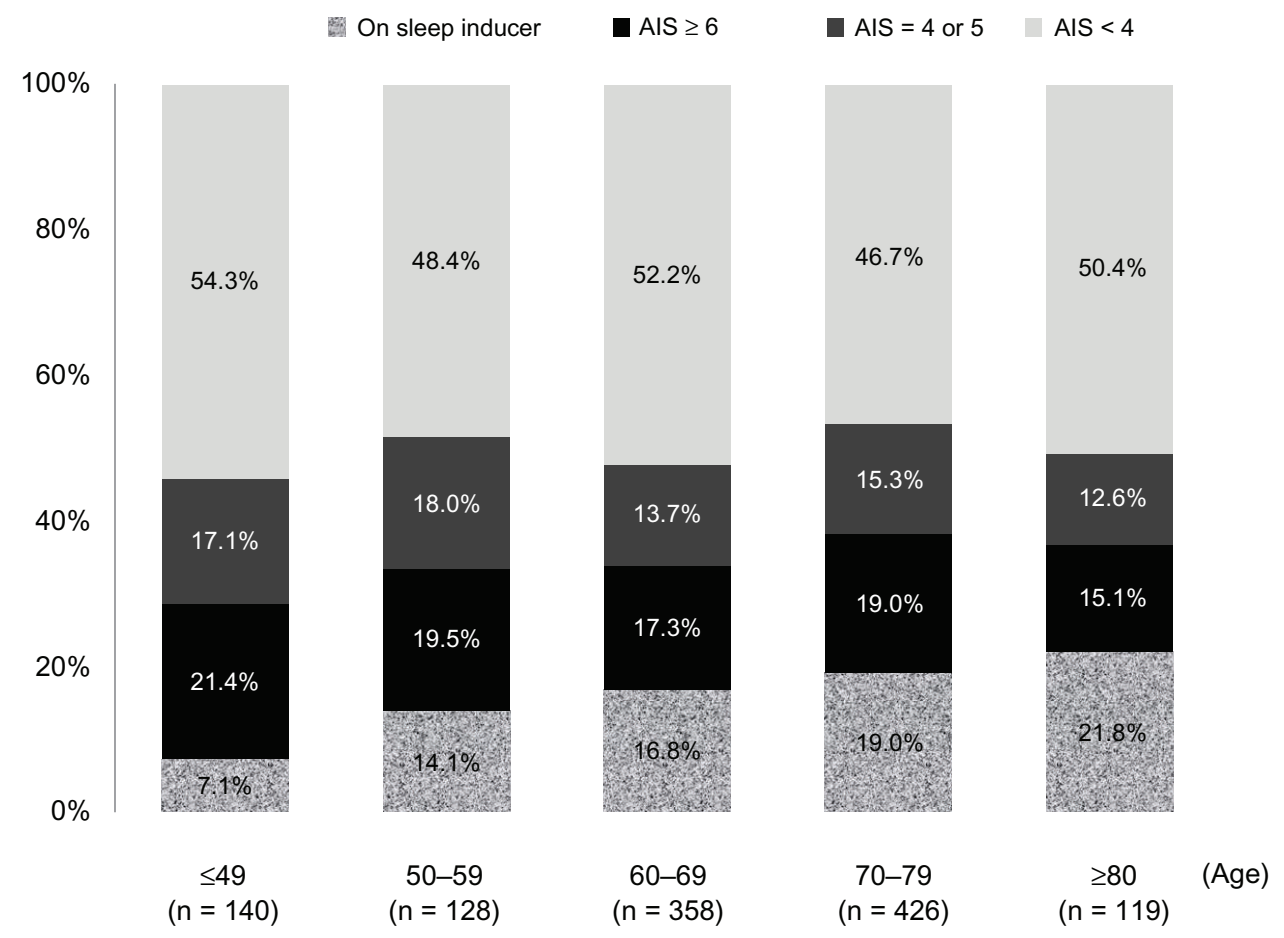

Figure 3 AIS by age.

Abbreviations: AIS, Athens Insomnia Scale; n, number.

corresponding odds ratio per unit score change was estimated at 1.5 (Figure 7).

\section{Discussion}

In the present study, our results showed that $16.8 \%$ of outpatients treated at urology departments were already using sleep inducers. This suggested that, when combining these patients with suspected cases of sleep disorders characterized by an AIS score of $\geq 4,51.5 \%$ of patients (or one in two patients) might have developed a sleep disorder as a complication of their disease. This figure exceeded that reported in a previous nationwide study examining the incidence of insomnia complaints in the general population among 3030 subjects who were aged $\geq 20$ years, where approximately $30 \%$ of subjects aged $\geq 60$ years were reported as having a sleep disorder as a complication. ${ }^{1}$

It is possible that sleep disorders may have been observed in comparatively higher percentages of patients treated at urology departments across all age groups because they have some kind of LUTS. However, the present study did not address the issue of medical complications, which may also cause sleep disorders, and thus further investigations are warranted.

A significant increase in the total IPSS score was observed in patients with moderate (a 2.3-fold increase) and severe symptoms (a 4.8-fold increase), compared to the score of

Dependent variable: state of sleep disorders (AIS $\geq 4$ )

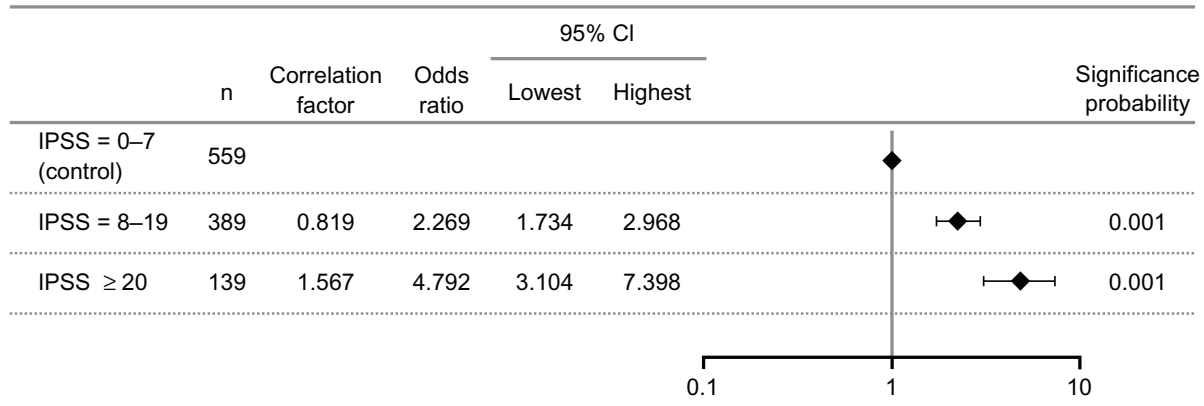

Figure 4 Analysis by logistic regression model.

Abbreviations: AIS, Athens Insomnia Scale; n, number; Cl, confidence interval; IPSS, International Prostate Symptom Score. 
Below cutoff value/equal or greater than cutoff value

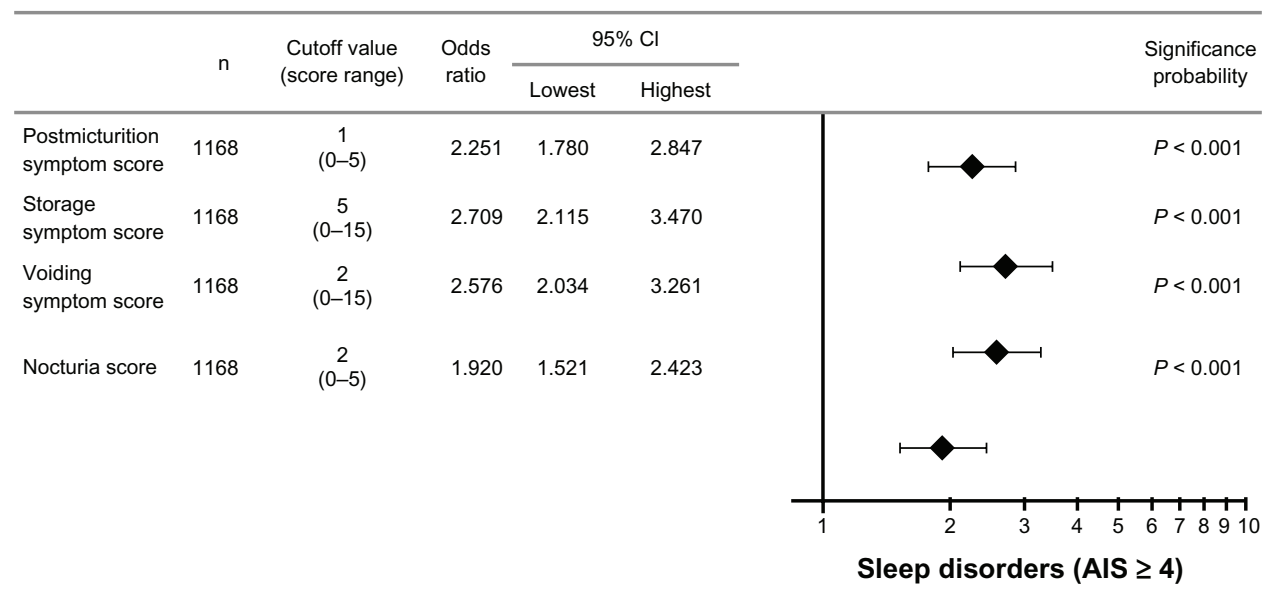

Figure 5 Odds ratio by subscore.

Abbreviations: $\mathrm{n}$, number; $\mathrm{Cl}$, confidence interval; AIS, Athens Insomnia Scale.

those with mild symptoms. This suggests that there is an association of more severe LUTS with a higher risk of sleep disorders.

Many studies have reported a correlation between sleep disorders and nocturia. ${ }^{3-9}$ Nocturnal awakening due to nocturia is four times more common than awakening due to pain, which establishes nocturia as a predictor of insomnia and as a factor that influences sleep quality. ${ }^{7}$ The present study indicates that, in addition to nocturia, other IPSS subscores may act as independent risk factors of sleep disorders. This is demonstrated by the observed 2.3-fold increase in the risk for suspected sleep disorders among patients with a postmicturition symptom subscore of $\geq 1$, the 2.7 -fold increase in risk among patients with a storage symptom subscore of $\geq 5$, and the 2.6-fold increase among patients with a voiding symptom subscore of $\geq 2$.

Although the mechanism behind the association between sleep disorders and these symptoms is not clear, long-term insomnia and nocturia are often found to be intricately associated in the elderly. While poor sleep quality may increase arousal during sleep, which results in secondary nocturia, prostatic hyperplasia in men has been reported to cause nocturia, ${ }^{16,17}$ which in turn leads to a sleep disorder. The present study found a positive correlation between nocturia and post-micturition, storage, and voiding symptom subscores. The severity of LUTS positively correlated with the frequency of nocturia, which may cause sleep disorders. Studies focusing solely on BPH

Below cutoff value/equal or greater than cutoff value

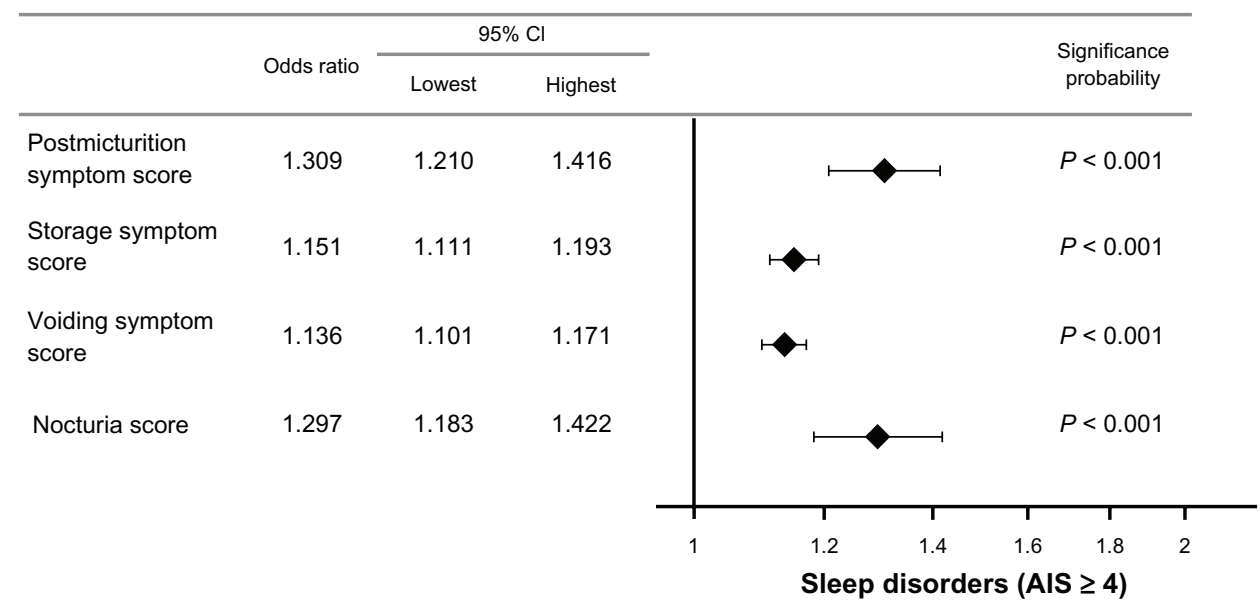

Figure 6 Odds ratio per unit of change in score.

Abbreviations: $\mathrm{Cl}$, confidence interval; AIS, Athens Insomnia Scale. 
Below cutoff value/equal or greater than cutoff value

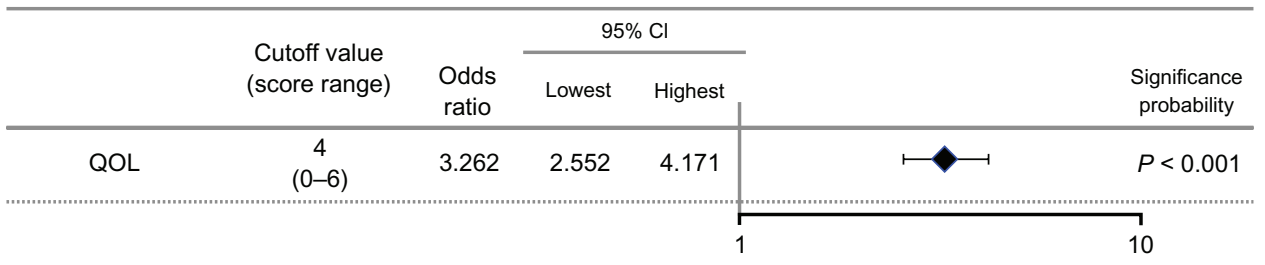

Odds ratio per unit score change

\begin{tabular}{|c|c|c|c|c|}
\hline & \multirow[b]{2}{*}{$\begin{array}{l}\text { Odds } \\
\text { ratio }\end{array}$} & \multicolumn{2}{|c|}{$95 \% \mathrm{Cl}$} & \multirow[b]{2}{*}{$\begin{array}{l}\text { Significance } \\
\text { probability }\end{array}$} \\
\hline & & Lowest & Highest & \\
\hline \multirow[t]{2}{*}{ QOL } & 1.463 & 1.359 & 1.576 & $P<0.001$ \\
\hline & & & & 10 \\
\hline
\end{tabular}

Figure 7 Association between IPSS, QOL, and sleep disorders.

Abbreviations: QOL, quality of life; $\mathrm{Cl}$, confidence interval; AIS, Athens Insomnia Scale.

have suggested that sleep disorders are caused not only by nocturia, but also by storage and voiding symptoms. The mechanism underlying the development of such sleep disorders may also involve imbalances in steroid hormone action, sympathetic nervous system activity, and immune dysfunction, which have been considered to potentially contribute to the development of symptomatic BPH and bladder outlet obstruction..$^{18}$

Some studies have also reported that disruption of the circadian rhythm may contribute to sleep disorders. ${ }^{19,20}$ The body's biological clock is involved in regulating bladder function and the amount of urine to be stored over the course of 24 hours, which is important for maintaining secure and sound sleep. Therefore, this mechanism may be involved in the association between LUTS and sleep disorders. ${ }^{21}$

Although sleep disorders are highly common among patients treated at urology departments, they have not been carefully studied yet. However, treating these sleep disorders will help maintain patients' level of activity and improve their QOL. It is also possible that improving LUTS may help improve sleep disorders. Therapeutic intervention involving a large number of cases is needed to further understand the association between LUTS and sleep disorders.

\section{Disclosure}

The authors report no conflicts of interest in this work. The authors of the present paper have no financial or commercial interests to disclose.

\section{References}

1. Kim K, Uchiyama M, Okawa M, Liu X, Ogihara R. An epidemiological study of insomnia among the Japanese general population. Sleep. 2000;23(1):41-47.

2. Kaneita Y, Uchiyama M, Yoshiike N, Ohida T. Associations of usual sleep duration with serum lipid and lipoprotein levels. Sleep. 2008;31(5):645-652.

3. Kageyama T, Kabuto M, Nitta H, et al. Prevalence of nocturia among Japanese adults. Psychiatry Clin Neurosci. 2000;54(3):299-300.

4. Asplund R. Nocturia in relation to sleep, somatic diseases and medical treatment in the elderly. BJU Int. 2002;90(6):533-536.

5. Yoshimura K, Oka Y, Kamoto T, Yoshimura K, Ogawa O. Differences and associations between nocturnal voiding/nocturia and sleep disorders. BJU Int. 2010;106(2):232-237.

6. Middelkoop HA, Smide-van den Doel DA, Neven AK, Kamphuisen HA, Springer CP. Subjective sleep characteristics of 1,485 males and females aged 50-93: effects of sex and age, and factors related to selfevaluated quality of sleep. J Gerontol A Biol Sci Med Sci. 1996;51(3): M108-M115.

7. Bliwise DL, Foley DJ, Vitiello MV, Ansari FP, Ancoli-Israel S, Walsh JK. Nocturia and disturbed sleep in the elderly. Sleep Med. 2009; 10(5):540-548.

8. Gopal M, Sammel MD, Pien G, et al. Investigating the associations between nocturia and sleep disorders in perimenopausal women. J Urol. 2008;180(5):2063-2067.

9. Coyne KS, Zhou Z, Bhattacharyya SK, Thompson CL, Dhawan R, Versi E. The prevalence of nocturia and its effect on health-related quality of life and sleep in a community sample in the USA. BJU Int. 2003;92(9):948-954.

10. Homma Y, Kakizaki H, Gotoh M, Takei M, Yamanishi T, Hayashi K. Epidemiologic survey on lower urinary tract symptoms in Japan. Nippon Hainyokino Gakkaishi. 2003;14:266-277. Japanese.

11. Nakagawa H, Niu K, Hozawa A, et al. Impact of nocturia on bone fracture and mortality in older individuals: a Japanese longitudinal cohort study. J Urol. 2010;184(4):1413-1418.

12. Kupelian V, Fitzgerald MP, Kaplan SA, Norgaard JP, Chiu GR, Rosen RC. Association of nocturia and mortality: results from the Third National Health and Nutrition Examination Study. J Urol. 2011;185(2):571-577.

13. Soldatos CR, Dikeos DG, Paparrigopoulos TJ. Athens Insomnia Scale: validation of an instrument based on ICD-10 criteria. J Psychosom Res. 2000;48(6):555-560. 
14. Soldatos CR, Dikeos DG, Paparrigopoulos TJ. The diagnostic validity of the Athens Insomnia Scale. J Psychosom Res. 2003;55(3):263-267.

15. American Academy of Sleep Medicine. International Classification of Sleep Disorders: Diagnostic and Coding Manual. 2nd ed. Rochester, MN: Sleep Disorders Association; 2005;(ICSD-2).

16. Hunter DJ, Berra-Unamuno A, Martin-Gordo A. Prevalence of urinary symptoms and other urological conditions in Spanish men 50 years old or older. J Urol. 1996;155(6):1965-1970.

17. Blanker MH, Bohnen AM, Groeneveld FP, Bernsen RM, Prins A, Ruud Bosch JL. Normal voiding patterns and determinants of increased diurnal and nocturnal voiding frequency in elderly men. $J$ Urol. 2000;164(4):1201-1205.
18. Cakir OO, McVary KT. LUTS and sleep disorders: emerging risk factor. Curr Urol Rep. 2012;13(6):407-412.

19. Zee PC, Mehta R, Turek FW, Blei AT. Portacaval anastomosis disrupts circadian locomotor activity and pineal melatonin rhythms in rats. Brain Res. 1991;560(1-2):17-22.

20. Steindl PE, Finn B, Bendok B, Rothke S, Zee PC, Blei AT. Disruption of the diurnal rhythm of plasma melatonin in cirrhosis. Ann Intern Med. 1995;123(4):274-277.

21. Negoro H, Kanematsu A, Doi M, et al. Involvement of urinary bladder Connexin43 and the circadian clock in coordination of diurnal micturition rhythm. Nat Commun. 2012;3:809.

\section{Publish your work in this journal}

Nature and Science of Sleep is an international, peer-reviewed, open access journal covering all aspects of sleep science and sleep medicine, including the neurophysiology and functions of sleep, the genetics of sleep, sleep and society, biological rhythms, dreaming, sleep disorders and therapy, and strategies to optimize healthy sleep. The journal welcomes

\section{Dovepress}

original research, clinical \& epidemiological studies, reviews \& evaluations, case reports and extended reports. The manuscript management system is completely online and includes a very quick and fair peerreview system, which is all easy to use. Visit http://www.dovepress.com/ testimonials.php to read real quotes from published authors.

\footnotetext{
Submit your manuscript here: http://www.dovepress.com/nature-and-science-of-sleep-journal
} 